\title{
36 DOES ACR/EULAR REMISSION MEAN IMPROVED FUNCTIONAL ABILITY, QUALITY OF LIFE AND WORK PRODUCTIVITY?
}

A M Patel, ${ }^{1}$ C L Amity, ${ }^{2}$ L M Frydrych, ${ }^{2}$ D M Jones, ${ }^{2}$ D Goudeau, ${ }^{2}$ H F Eng, ${ }^{2}$ D Kyle, ${ }^{2}$ M. Saul, ${ }^{2}$ D H Solomon, ${ }^{3}$ S R Wisniewski, ${ }^{2}$ L W Moreland, ${ }^{2}$ M C Levesque ${ }^{2}$ (and the RACER physicians group) ' University of Pittsburgh/Children's Hospital of Pittsburgh of UPMC, Pittsburgh, PA, USA; ${ }^{2}$ University of Pittsburgh, Pittsburgh, PA, USA; ${ }^{3}$ Brigham \& Womens Hospital, Boston, MA, USA

10.1136/annrheumdis-2011-201230.36

Background/purpose RA patients meeting the new American College of Rheumatology/European League Against Rheumatism (ACR/EULAR) definition of remission should have better clinical outcomes than those with low disease activity. Therefore, the authors compared functional ability, health-related quality of life (HROOL), work impairment and medication use between those meeting new ACR/EULAR remission and those in low disease activity or other defined states of remission.

Method Subjects were from the University of Pittsburgh Rheumatoid Arthritis Comparative Effectiveness Research (RACER) registry ( $\mathrm{n}=721)$. The authors analysed self-administered patient questionnaires (routine assessment of patient index-3 (RAPID3)), short form-12 (SF12), work productivity and activity impairment (WPAI)), physician exam data (disease activity score-28 joint count (DAS28) and clinical disease activity index (CDAI)) from a single RACER visit. Subjects were grouped according to whether they met ACR/EULAR remission, DAS28 and CDAI remission, low, moderate and high disease activity. Demographics, questionnaire data and medication use were compared between those who did not fulfil the new remission criteria but did fulfil other remission and low disease activity states using Mann-Whitney $U$ and Pearson $\chi^{2}$ tests.

Result ACR/EULAR remission was achieved by $17.6 \%$ of the cohort. Age, sex, race and disease duration were similar between 
Table 1 Comparison of different remission and low disease activity definitions to the ACR/EULAR definition of remission and their effect on work productivity and health assessment $(n=721)$

\begin{tabular}{|c|c|c|c|c|c|c|c|}
\hline & $\begin{array}{l}\text { ACR/EULAR } \\
\text { remission }(n=127)\end{array}$ & $\begin{array}{l}\text { DAS28 remission } \\
(n=147)\end{array}$ & $\mathbf{p}$ & $\begin{array}{l}\text { DAS28 low disease } \\
\text { activity }(n=125)\end{array}$ & $\mathbf{p}$ & $\begin{array}{l}\text { CDAI low disease } \\
\text { activity }(n=246)\end{array}$ & $\mathbf{p}$ \\
\hline SF12 PCS & $45.8 \pm 9.4$ & $38.4 \pm 9.1$ & $<0.0001$ & $34.4 \pm 8.9$ & $<0.0001$ & $36.76 \pm 9.3$ & $<0.0001$ \\
\hline MCS & $52.4 \pm 8.1$ & $48.8 \pm 10.0$ & 0.005 & $47.0 \pm 9.4$ & $<0.0001$ & $48.0 \pm 9.9$ & $<0.0001$ \\
\hline WPAI (\%) Act imp & $13.7 \pm 20.7$ & $29.4 \pm 26.3$ & $<0.0001$ & $42.6 \pm 24.2$ & $<0.0001$ & $35.1 \pm 26.6$ & $<0.0001$ \\
\hline Time missed & $0.6 \pm 3.2$ & $9.6 \pm 23.7$ & 0.01 & $2.7 \pm 11.4$ & 0.143 & $5.9 \pm 17.8$ & 0.011 \\
\hline Imp working & $9.4 \pm 18.5$ & $20.0 \pm 21.9$ & $<0.0001$ & $29.3 \pm 25.2$ & $<0.0001$ & $24.5 \pm 22.8$ & $<0.0001$ \\
\hline Overall Imp & $10.1 \pm 18.7$ & $25.8 \pm 29.4$ & $<0.0001$ & $31.5 \pm 26.6$ & $<0.0001$ & $28.0 \pm 26.8$ & $<0.0001$ \\
\hline RAPID3 & $1.0 \pm 1.0$ & $1.9 \pm 1.7$ & $<0.0001$ & $3.4 \pm 1.8$ & $<0.0001$ & $3.0 \pm 1.8$ & $<0.0001$ \\
\hline
\end{tabular}

Remission definitions were individually applied to all subjects and those fulfilling ACR/EULAR remission are removed from other categories so same subjects are not represented in multiple groups. Majority of those in CDAl remission fulfilled ACR/EULAR remission (106/109) so comparisons were not made. RACER: Rheumatoid Arthritis Comparative Effectiveness Research ACR/EULAR=American College of Rheumatology/ European League Against Rheumatism; SF12: short form-12; PCS: physical component score; MCS: mental component score; WPAI: Work Productivity and Activity Impairment; RAPID3: Routine Assessment of Patient Index Data-3.

disease activity groups. The mean SF-12 physical and mental component scores (PCS/MCS) for subjects in ACR/EULAR remission were similar to healthy, comparably aged subjects. Subjects in CDAI remission $(\mathrm{n}=109)$ also met ACR/EULAR remission and had comparable SF-12, WPAI and RAPID3 scores (data not shown). In contrast, not all subjects in DAS28 remission $(n=274)$ also met the ACR/EULAR definition of remission $(\mathrm{n}=127)$. Subjects in DAS28 remission but not ACR/EULAR remission ( $\mathrm{n}=147)$ and subjects in DAS28 and CDAI low disease activity had worse SF12, WPAI and RAPID3 scores (table 1). The use of DMARDs and/or biologics was similar between the remission and low disease activity groups. However, the ACR/ EULAR and CDAI remission groups used significantly less opiates and corticosteroids than subjects in DAS remission and all other disease activity groups $(\mathrm{p}<0.0001)$.

Conclusion RA subjects in ACR/EULAR remission had a quality of life similar to healthy comparably aged subjects suggesting that the new ACR/EULAR remission criteria represent a true state of remission. The DAS28 definition of remission is not comparable since RA subjects in ACR/EULAR remission had better RAPID3, SF12 and WPAI scores. ACR/ EULAR remission was associated with improved functional ability, HRQOL and work productivity compared to DAS28 and CDAI low disease activity. Medication data suggests that for subjects not in remission, achieving remission will require more optimal and/or greater use of combinations of immunosuppressive therapies. Our future goal is to determine the cost-effectiveness and the risk:benefit of more aggressive treatment approaches for patients not yet in remission and to study remission longitudinally. 